\title{
The Transit Light Curve Project. Viii. Six Occultations of the Exoplanet Tres-3
}

\section{Citation}

Winn, Joshua N., Matthew J. Holman, Avi Shporer, José Fernández, Tsevi Mazeh, David W. Latham, David Charbonneau, and Mark E. Everett. 2008. "THE TRANSIT LIGHT CURVE PROJECT. VIII. SIX OCCULTATIONS OF THE EXOPLANET TrES-3." The Astronomical Journal 136 (1): 267-71. https://doi.org/10.1088/0004-6256/136/1/267.

\section{Permanent link}

http://nrs.harvard.edu/urn-3:HUL.InstRepos:41417348

\section{Terms of Use}

This article was downloaded from Harvard University's DASH repository, and is made available under the terms and conditions applicable to Other Posted Material, as set forth at http:// nrs.harvard.edu/urn-3:HUL.InstRepos:dash.current.terms-of-use\#LAA

\section{Share Your Story}

The Harvard community has made this article openly available. Please share how this access benefits you. Submit a story.

Accessibility 


\title{
THE TRANSIT LIGHT CURVE PROJECT. VIII. SIX OCCULTATIONS OF THE EXOPLANET TrES-3
}

\author{
Joshua N. Winn ${ }^{1}$, Matthew J. Holman ${ }^{2}$, Avi ShPorer ${ }^{3}$, José Fernández ${ }^{2}$, Tsevi Mazeh ${ }^{3}$, David W. Latham ${ }^{2}$, \\ David Charbonneau ${ }^{2}$, and Mark E. Everett ${ }^{4}$ \\ ${ }^{1}$ Department of Physics, and Kavli Institute for Astrophysics and Space Research, Massachusetts Institute of Technology, Cambridge, MA 02139, USA \\ ${ }^{2}$ Harvard-Smithsonian Center for Astrophysics, 60 Garden Street, Cambridge, MA 02138, USA \\ ${ }^{3}$ Wise Observatory, Tel Aviv University, Tel Aviv 69978, Israel \\ ${ }^{4}$ Planetary Science Institute, 1700 E. Fort Lowell Rd., Suite 106, Tucson, AZ 85719, USA \\ Received 2008 February 18; accepted 2008 April 15; published 2008 June 10
}

\begin{abstract}
We present photometry of the exoplanet host star TrES-3 spanning six occultations (secondary eclipses) of its giant planet. No flux decrements were detected, leading to $99 \%$-confidence upper limits on the planet-to-star flux ratio of $2.4 \times 10^{-4}, 5.0 \times 10^{-4}$, and $8.6 \times 10^{-4}$ in the $i-, z^{-}$, and $R$-bands respectively. The corresponding upper limits on the planet's geometric albedo are 0.30, 0.62, and 1.07. The upper limit in the $i$-band rules out the presence of highly reflective clouds, and is only a factor of 2-3 above the predicted level of thermal radiation from the planet.
\end{abstract}

Key words: planetary systems - stars: individual (TrES-3, GSC 0308900929) - techniques: photometric

Online-only material: machine-readable and VO tables

\section{INTRODUCTION}

The detection of reflected light from close-in giant planets is a difficult but worthwhile goal. Knowledge of the albedo is important because the stellar insolation is a critical factor in the atmospheric structure and the overall thermal balance of these planets (Guillot et al. 1996; Saumon et al. 1996; Seager \& Sasselov 1998). Measurement of the planet's reflection spectrum, or at least the broad wavelength dependence of the albedo, would provide clues about the dominant scattering mechanisms and constituents in the planetary atmosphere (see, e.g., Seager \& Sasselov 1998; Marley et al. 1999; Sudarsky et al. 2000; Barman et al. 2001). The difficulty is that the reflected light is a very small fraction of the direct starlight, producing a planet-to-star flux ratio at the opposition of $\epsilon_{\lambda}=$ $p_{\lambda}\left(R_{p} / a\right)^{2}$, where $R_{p}$ is the planetary radius, $a$ is the orbital separation, and $p_{\lambda}$ is the wavelength-dependent geometric albedo. ${ }^{5}$ Even for a planet as large as Jupiter, and an orbital separation as small as $0.05 \mathrm{AU}, \epsilon_{\lambda} \approx 10^{-4} p_{\lambda}$. The reflected signal is also expected to vary over the orbital period, in a manner depending on the orbital inclination and the phase function of the planetary atmosphere.

Early attempts to detect reflected light from close-in giant planets, by Charbonneau et al. (1999), Cameron et al. (1999, 2002), and Leigh et al. (2003a, 2003b), relied on optical spectroscopy. Those investigators sought the reflected copies of the stellar spectral lines by combining suitably Doppler-shifted spectra taken over a range of orbital phases. They did not detect the reflected signal and placed upper limits ${ }^{6}$ on the geometric albedo in the visual band of $\sim 0.1-0.5$, subject to some ambiguity because of the unknown radius and orbital inclination of the planets they observed. Liu et al. (2008) recently revisited the spectroscopic technique and applied it to HD 209458, for which the planetary radius and orbital inclination are known, but the

\footnotetext{
5 The geometric albedo is defined as the flux reflected by the planet when viewed at opposition (full phase), divided by the flux that would be reflected by a flat and perfectly diffusing surface with the same cross-sectional area as the planet.

6 Although Cameron et al. (1999) reported a detection with greater than $95 \%$ confidence, additional data obtained by the same group did not confirm the detection; see Cameron et al. (2002).
}

available data provided only a weak constraint on the geometric albedo $(0.8 \pm 1.6$ from $554-681 \mathrm{~nm})$.

Another detection method involves polarimetry. The Stokes parameters are expected to vary over the planetary orbital period because reflected light is preferentially polarized while direct starlight is unpolarized (see, e.g., Hough et al. 2006). Berdyugina et al. (2008) reported a detection of a time-variable polarized signal from HD 189733. Taking the scattering radius to be the same as the optical radius measured through transit photometry, the implied geometric albedo is larger than $2 / 3$, which is the geometric albedo of a perfectly diffusing sphere. While this is physically possible, and indeed some solar system objects have geometric albedos exceeding unity due to strong backscattering, Berdyugina et al. (2008) preferred an interpretation in which the albedo is smaller and the scattering radius is somehow larger than the optical radius measured during transits.

For planets whose orbits are viewed close enough to edge-on that they undergo periodic occultations by the star, a powerful and conceptually simple method is available: when the planet is hidden by the star, the total light should diminish by the fraction $\epsilon_{\lambda}$. Edge-on systems are also advantageous because the planetary radius and orbital inclination can be determined precisely from transit observations. ${ }^{7}$ If the measurement noise were limited only by photon statistics, the photometric technique could be employed with a smaller telescope than would be needed for either the spectroscopic or polarimetric techniques. However, ground-based photometry is generally afflicted by systematic errors that prevent one from achieving the required precision. To date, only spaceborne photometry has provided meaningful upper limits on exoplanetary albedos. In particular, Rowe et al. (2007) have used the Microvariability and Oscillations of Stars (MOST) satellite to set a $3 \sigma$ upper limit of 0.17 on the geometric albedo of HD 209458.

Discoveries of transiting planets have abounded in the past few years, and there are now several systems known with larger values of $\left(R_{p} / a\right)^{2}$. One of the most favorable systems

\footnotetext{
7 It is possible, however, for a planet on an eccentric orbit to exhibit occultations without transits, or transits without occultations (see, e.g., Irwin et al. 2008).
} 
is TrES-3, discovered by O'Donovan et al. (2007), for which $\left(R_{p} / a\right)^{2}=7.5 \times 10^{-4}$. In this system, a planet with mass $2 M_{\mathrm{Jup}}$ and radius $1.3 R_{\text {Jup }}$ circles a G dwarf star with an orbital period of $31 \mathrm{~h}$. This paper relates our attempts to detect reflected light from the TrES-3 planet using ground-based photometry with meterclass telescopes. In Section 2, we describe our observations and data reduction procedures. In Section 3, we present our results. In Section 4, we place these results in the context of other observations and of theoretical expectations for the albedo and the thermal emission spectrum.

\section{OBSERVATIONS AND DATA REDUCTION}

We observed TrES-3 on six different nights when planetary occultations were expected, according to the ephemeris of O'Donovan et al. (2007). Assuming that the planetary orbit is circular, as expected for a planet with such a short orbital period, ${ }^{8}$ the uncertainty in the predicted mid-occultation time was smaller than 3 min on each night. Observations on other nights were attempted, but only the six nights described below offered clear enough skies for high-precision photometry.

On 2007 April 27, 2007 July 4, 2007 September 14, and 2008 March 12, we used the $1.2 \mathrm{~m}$ telescope at the Fred L. Whipple Observatory (FLWO) on Mount Hopkins, Arizona. (Here and elsewhere, the quoted date refers to the UT date at the start of the night.) The detector was KeplerCam, a $4096^{2}$ CCD with a square field of view 23.'1 on a side (Szentgyorgyi et al. 2005). We binned the images $2 \times 2$, giving a scale of 0 '. 68 per binned pixel. We obtained repeated $60 \mathrm{~s}$ exposures over the course of 4-5 $\mathrm{h}$ bracketing the predicted mid-occultation time. The dead time between exposures was $11 \mathrm{~s}$. Autoguiding maintained the pointing to within 2-3 pixels throughout the observations. On the first night, 2007 April 27, we observed through a Sloan $i$ filter $(\approx 0.7-0.85 \mu \mathrm{m})$, as the star rose from an air mass of 1.4 to the meridian. The full width at half-maximum (FWHM) of stellar images was about 4.5 pixels $\left(3^{\prime \prime}\right)$. On the next two nights, 2007 July 4 and 2007 September 14, we observed through a Sloan $z$ filter $(\approx 0.85-1.0 \mu \mathrm{m}$, with the red cutoff arising from the quantum efficiency of the detector). On both of those nights, the target star began near the meridian and set to an air mass near 2.0 throughout the observations, and the FWHM was approximately 3 pixels $\left(2^{\prime \prime}\right)$. On the last night, 2008 March 12, we chose the Sloan $i$ filter again and observed through an air mass ranging from 1.1 to 2.0. Conditions were nearly photometric, and the seeing was exceptionally good and stable, with an FWHM of 2.1 pixels $(1 " .4)$

On UT 2007 August 26 and 2007 October 3, we used the $1 \mathrm{~m}$ telescope at Wise Observatory in Israel. We used a Princeton Instruments $1340 \times 1300$ back-illuminated CCD, giving a field of view of $13.0 \times 12.6$ and a pixel scale of $0{ }^{\prime \prime} .58$. The readout and refresh time was $25 \mathrm{~s}$, and we used exposure times ranging from $60 \mathrm{~s}$ to $160 \mathrm{~s}$, depending on the air mass and seeing. The guider generally maintained the pointing to within a few pixels, but there was a glitch on each night that led to a 10-20 pixel offset. We observed through a Bessell $R$ filter $(\approx 0.55-0.7 \mu \mathrm{m})$. On August 26, we observed for $6 \mathrm{~h}$ as the target star set from the meridian to an air mass of 2.8 , and on October 3 , we observed for $4 \mathrm{~h}$ as the target set from air mass 1.1 to 2.5. In both cases,

\footnotetext{
8 The timescale for tidal circularization is $\sim 2 \times 10^{7} \mathrm{yr}$, using Equation (9) of Rasio et al. (1996) with the system parameters of O'Donovan et al. (2007) and a tidal quality factor $Q=10^{5}$. This is shorter than the estimated age of the star, $5 \times 10^{8}$ yr (Torres et al. 2008). The current radial velocity data are consistent with a circular orbit and give an upper limit on the eccentricity of 0.12 with $99 \%$ confidence.
}

Table 1

Photometry of TrES-3

\begin{tabular}{lccc}
\hline \hline Telescope & Filter & Heliocentric Julian date & Relative flux \\
\hline FLWO & $i$ & 2454217.806989 & 0.99905 \\
FLWO & $i$ & 2454217.807834 & 1.00095 \\
FLWO & $i$ & 2454217.808667 & 1.00006 \\
FLWO & $i$ & 2454217.809524 & 1.00156 \\
FLWO & $i$ & 2454217.810369 & 1.00144 \\
FLWO & $i$ & 2454217.811214 & 0.99904
\end{tabular}

Notes. The time stamps represent the Heliocentric Julian date at the time of mid-exposure.

(This table is available in its entirety in machine-readable and Virtual Observatory (VO) forms in the online journal. A portion is shown here for guidance regarding its form and content.)

the data obtained through an air mass greater than 2.0 proved to be much noisier than the rest of the data, and ultimately were not used in our analysis. On both nights the seeing steadily worsened with increasing air mass, from an FWHM of 2.5 pixels to 4.5 pixels ( 1 ". 8 to 3 ". 2 ).

We used standard IRAF procedures for overscan correction, trimming, bias subtraction, and flat-field division. We performed aperture photometry of TrES-3 and several nearby stars. The sum of the fluxes of the comparison stars was taken to be the comparison signal. The flux time series for TrES-3 was divided by the comparison signal, and then by a constant chosen to give a unit mean flux outside the predicted occultation. We experimented with different choices for the aperture size, and different combinations of the comparison stars, aiming to minimize the standard deviation of the out-of-occultation portion of the TrES-3 light curve. Not surprisingly, the best results were obtained when the aperture diameter was about twice as large as the FWHM of the stellar images, and when as many comparison stars as possible were used with mean fluxes ranging from $50 \%$ to $150 \%$ of the mean flux of TrES-3. For the FLWO data, 10-13 stars were used, and for the Wise data, 6-7 stars were used. The Wise light curve had an abrupt jump at precisely the time when the guiding failed and the pointing changed; to correct for this, we set the mean out-of-occultation flux to be unity for each of the two segments of data. Finally, for all data sets, we performed an air-mass correction. Using only the out-of-occultation data, we fitted the light curve to an exponential function of air mass, divided by the best-fitting function, and renormalized the light curve to have unit mean flux outside the occultation.

The final relative photometry is plotted in Figure 1, and the data are given in numerical form in Table 1. One of the transit light curves of O'Donovan et al. (2007) is also plotted in Figure 1, because the transit data were used in our model for the occultation data, as described in the next section. The duration of the occultation is expected to be nearly the same as the duration of the transit, since the orbit is presumed to be circular, as discussed further in Section 4.

Since no flux decrement is obvious in any of the light curves, the results are upper limits on the planet-to-star flux ratio in each bandpass. This makes it especially important to understand the characteristics of the noise. The out-of-occultation FLWO $i$ light curves from 2007 April 26 and 2008 March 12 have standard deviations of $9.1 \times 10^{-4}$ and $9.0 \times 10^{-4}$, respectively. The expected level of photon-counting (Poisson) noise is $8 \times 10^{-4}$, and the expected level of scintillation noise is $3 \times 10^{-4}$, according to Equation (1) of Young (1967). The quadrature sum 

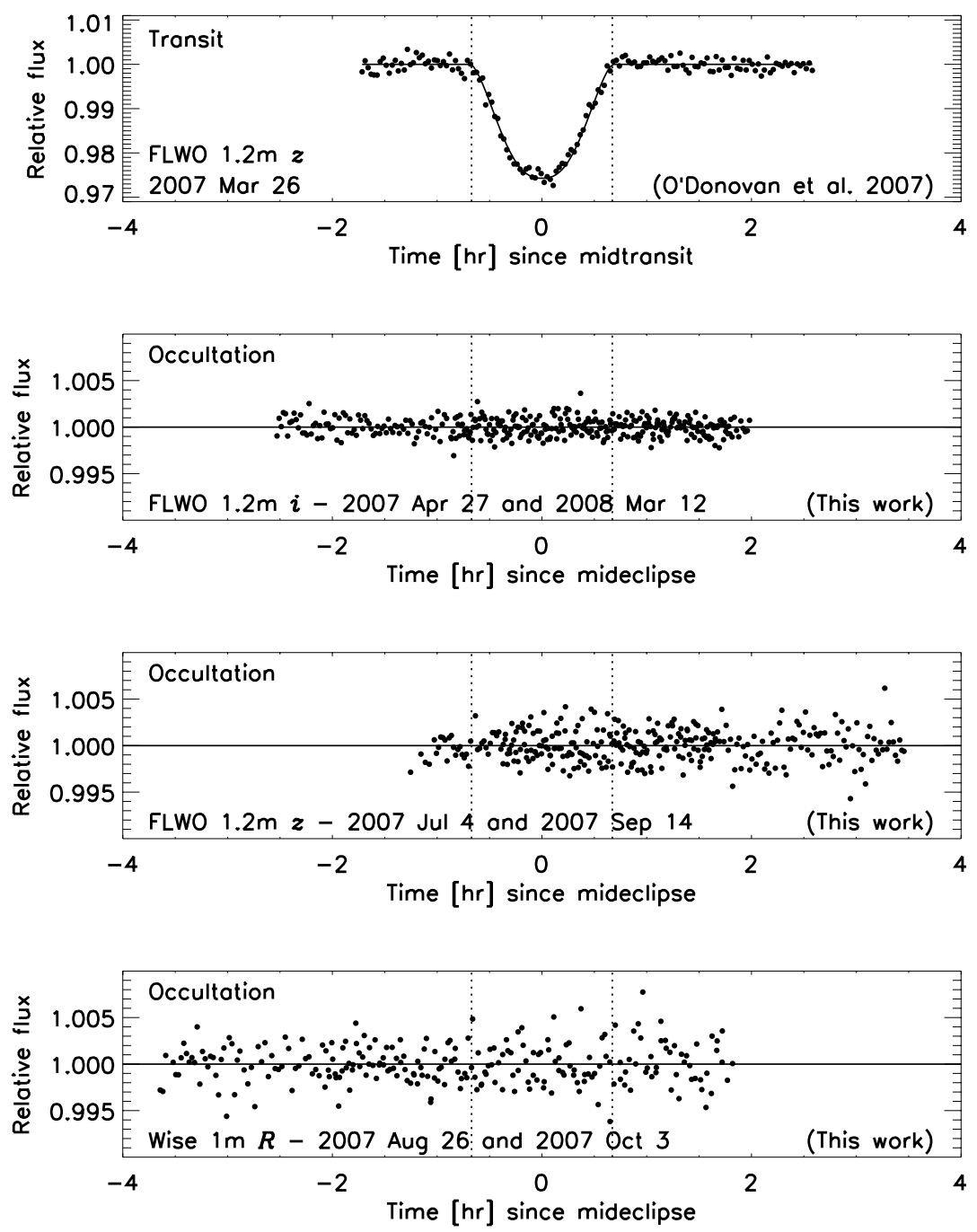

Figure 1. Photometry of TrES-3. The top panel shows the $z$-band transit photometry of O'Donovan et al. (2007). The other three panels show our photometry spanning occultations (secondary eclipses). The dotted lines show the expected start and end of the occultation.

of these terms is $8.5 \times 10^{-4}$. The FLWO $z$ light curves from 2007 July 14 and 2007 September 14 have standard deviations of $1.8 \times 10^{-3}$ and $1.4 \times 10^{-3}$, as compared to the expected level of about $1.2 \times 10^{-3}$ from Poisson and scintillation noise. Thus, most of the observed noise can be accounted for. The noise in the $i$-band light curves, in particular, is only 6\%-7\% larger than the noise from these two expected sources.

The out-of-occultation Wise data from 2007 August 26 and 2007 October 3 have standard deviations of $2.2 \times 10^{-3}$ and $1.9 \times 10^{-3}$. In both cases the expected level of photon-counting noise is about $10^{-3}$ and the expected level of scintillation noise is about $3 \times 10^{-5}$. Thus, the noise in the Wise data is about twice as large as one would expect from these two sources. Because of the jump that was observed in each light curve at the time of a large pixel shift, we suspect that at least some (and perhaps most) of the excess noise is due to an imperfect flat field. On other nights when the telescope lost tracking more frequently the noise level was indeed even higher, although those other nights were also characterized by poorer sky conditions, making it impossible to isolate the source of excess noise.

Next we tested for any correlations with time or other external variables, and for significant non-Gaussianity. We found no significant correlation (a correlation coefficient $<0.2$ ) between the final relative flux data and the air mass, the pixel position, or the measured shape parameters (FWHM, ellipticity, position angle) of stellar images. Of course, we had already decorrelated against the air mass, and for the Wise data we had already removed the effects of the two pointing glitches, so the lack of correlations with those variables was expected. For each light curve, we also examined the histogram of flux values, the autocorrelation function, and the factor by which the noise level is reduced when the data are binned. Because the histograms are approximately Gaussian, the autocorrelations are generally as small as would be expected for uncorrelated noise, and the noise falls approximately as $1 / \sqrt{N}$, in what follows we treat the noise as Gaussian and uncorrelated.

\section{DETERMINATION OF UPPER LIMITS ON THE ALBEDO}

One might imagine measuring the planet-to-star flux ratio, $\epsilon_{\lambda}$, by finding the ratio of the mean flux during the occultation to the mean out-of-occultation flux. However, because the TrES-3 planet is on a nearly-grazing trajectory, the durations of the ingress and egress cannot be ignored. Furthermore, the uncertainty in the orbital inclination $i$ introduces some uncertainty in the expected shape of the light curve, and the conversion from $\epsilon_{\lambda}$ into the geometric albedo $p_{\lambda}$ requires knowledge of the ratio $R_{p} / a$ and its uncertainty. 
To take all of these factors into account, we simultaneously fitted a parameterized model to our $R$-, $i$-, and $z$-band occultation photometry and the high-precision $B$ - and $z$-band transit photometry of O'Donovan et al. (2007). The model and the fitting method are similar to those we have employed in previous papers in this series (see, e.g., Winn et al. 2007; Holman et al. 2007). The model posits a circular orbit of a planet with radius $R_{p}$ and a star with radius $R_{\star}$, with orbital inclination $i$, orbital separation $a$, and period $P$. The flux ratio between the fullyilluminated disk of the planet and the full disk of the star is $\epsilon_{k}$, where $k$ refers to the bandpass. When the planet is projected in front of the star, or vice versa, we use the analytic formulae of Mandel \& Agol (2002) to compute the appropriate flux decrement. We assumed that the flux received from the planet does not vary appreciably over the $5 \mathrm{~h}$ span of our occultation data, i.e., that the planet's phase function is constant within $30^{\circ}$ of opposition. For the star, we assumed the limb-darkening law to be quadratic, with coefficients ${ }^{9}$ taken from Claret $(2000,2004)$. The model parameters were $\epsilon_{i}, \epsilon_{z}, \epsilon_{R}, R_{p} / a, R_{\star} / a, i, P$, and $T_{c}$ (a particular time of mid-transit), along with the air mass correction parameters for each light curve.

For the transit light curves, we adopted the same flux uncertainties as O'Donovan et al. (2007). For the occultation light curves, based on the investigation of the photometric errors described in the previous section, we took the standard deviation of the out-of-occultation flux to be the uncertainty in each flux measurement. To determine the best-fitting values of the parameters and their uncertainties, we used a Markov Chain Monte Carlo (MCMC) algorithm. This algorithm creates a random sequence of points in parameter space whose density is an approximation of the a posteriori probability distribution of the parameter values. The sequence is generated from an initial point by iterating a "jump function," which in our case was the addition of a Gaussian random number to a randomlychosen parameter. If the new point has a lower $\chi^{2}$ than the previous point, the jump is executed; if not, the jump is executed with probability $\exp \left(-\Delta \chi^{2} / 2\right)$. We used an isotropic prior for the orbital inclination (uniform in $\cos i$ ), and a Gaussian prior in the orbital period to enforce consistency with the value $P=1.30619 \pm 0.00001 \mathrm{~d}$, which was determined by O'Donovan et al. (2007) using the yearlong baseline between the TrES survey data and the high-precision light curves. We used uniform priors for all other parameters. The planet-to-star flux ratios were required to be positive. After creating several independent chains to verify that they all converged to the same region of parameter space, we created one long chain of $5 \times 10^{6}$ points for our final results.

In Table 2, we provide the results for the $99 \%$-confidence upper limit on the planet-to-star flux ratio and the geometric albedo for each bandpass. The geometric albedo was computed as $p_{\lambda}=\epsilon_{\lambda}\left(a / R_{p}\right)^{2}$. The upper limit was defined as the value for which the cumulative a posteriori probability distribution took the value 0.99 . Figure 2 shows the a posteriori probability distribution for the geometric albedo based on the data from each bandpass, and for all of the data in combination. The results

\footnotetext{
9 Specifically, we used $a=0.2508, b=0.3019$ for the $z$-band data; and $a=0.7250, b=0.0967$ for the $B$-band data. These are based on interpolations for a star with $T_{\text {eff }}=5720 \mathrm{~K}, \log g=4.6$, and solar metallicity. Southworth (2008) has found that the uncertainties in the transit parameters (the scaled radii of the star and planet, and the orbital inclination) are underestimated when the limb-darkening coefficients are fixed at theoretical values. However, our results for the geometric albedo are not susceptible to this problem, as we confirmed by repeating the fit with a linear limb-darkening law and allowing the coefficients to be free parameters.
}

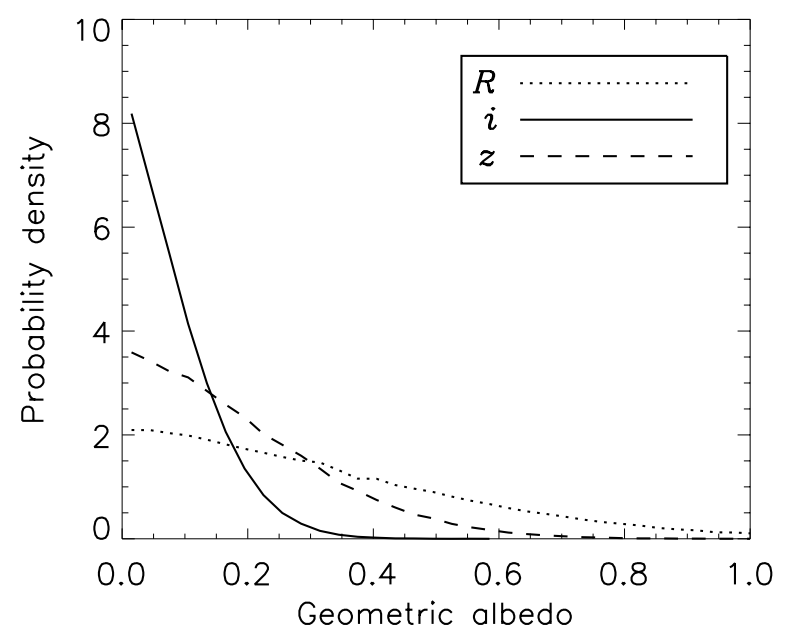

Figure 2. Constraints on the geometric albedo of exoplanet TrES-3, averaged over various bandpasses. Each curve shows the a posteriori probability distribution determined with the MCMC algorithm.

Table 2

Results of Occultation Photometry of TrES-3

\begin{tabular}{lcc}
\hline \hline Bandpass & \multicolumn{2}{c}{ Upper limit (99\% confidence) } \\
\cline { 2 - 3 } & Planet-to-star flux ratio & Geometric albedo \\
\hline$i$ & 0.00024 & 0.30 \\
$z$ & 0.00050 & 0.62 \\
$R$ & 0.00086 & 1.07 \\
\hline
\end{tabular}

for the transit-based parameters $R_{p} / R_{\star}, R_{\star} / a, i$, and $T_{c}$ were in agreement with the published values of O'Donovan et al. (2007).

\section{DISCUSSION AND SUMMARY}

This study has resulted in empirical upper limits on the planetto-star flux ratio, and the geometric albedo, in the wavelength range $0.6-1.0 \mu \mathrm{m}$. The most stringent upper limit was obtained in the Sloan $i$-band, where $p_{i}<0.30$ with $99 \%$ confidence. To put this result in perspective, we remind the reader that a perfectly-diffusing sphere has a geometric albedo of $2 / 3$. The TrES-3 planet is less reflective than such a sphere, at least for observations in the $i$ - and $z$-bands. The bandpass-averaged geometric albedos of Jupiter in the $R$-, $i$-, and $z$-bands are approximately $0.50,0.45$, and 0.25 , respectively (Karkoschka 1994). Our results show that TrES-3 is less reflective than Jupiter in the $i$-band, although they do not enable a meaningful comparison in the other two bands. Firm comparisons to other exoplanets are also difficult at this stage because almost all published reports are upper limits. Using the spectroscopic method, Charbonneau et al. (1999), Cameron et al. (2002), and Leigh et al. (2003a, 2003b) have placed upper limits of 0.1-0.4 on various systems, although in those cases the results are subject to extra uncertainty because the planetary radius and orbital inclinations are not known. Rowe et al. (2007) used several months of spaceborne photometry of HD 209458 to conclude $p_{\mathrm{opt}}<0.17$ for that system with $3 \sigma(99.73 \%)$ confidence, over an optical bandpass ranging from 0.4 to $0.7 \mu \mathrm{m}$. At the same confidence level of $99.73 \%$, our upper limit in the Sloan $i$-band is $p_{i}<0.35$. As mentioned in Section 1, Berdyugina et al. (2008) found a surprisingly large planet-to-star flux ratio for HD 189733, indeed large enough that those authors suggested the scattering radius is larger than the transit radius. Our results do not permit such an interpretation for TrES-3. 
Theoretical reflection spectra for strongly irradiated giant planets such as TrES-3 depend upon many factors and are especially sensitive to the presence or absence of clouds. Without highly reflective clouds, they are generally expected to have very low optical albedos. The underlying cause of the low albedo in cloud-free models is strong absorption in the pressure-broadened resonance doublet transitions of sodium and potassium (see, e.g., Seager et al. 2000; Marley et al. 1999; Sudarsky et al. 2000) or, for the hottest planets, the plentiful electronic transitions of gaseous $\mathrm{TiO}$ and $\mathrm{VO}$ (Hubeny et al. 2003; Sharp \& Burrows 2007; Fortney et al. 2007). Condensates may form reflective clouds that can drastically raise the albedo. The particular condensates that are available will depend on the temperature and pressure profile of the atmosphere.

As a specific example, Sudarsky et al. $(2000,2003)$ predicted that gas giants with effective temperatures between about $900 \mathrm{~K}$ and $1500 \mathrm{~K}$ ("Class IV" in their terminology) have optical albedos $\lesssim 0.2$ in the bandpasses we employed, while for temperatures $\lesssim 1500 \mathrm{~K}$ ("Class V"), the albedo is as large as 0.6 . The difference is that the hotter planets have a high layer of opaque silicate and iron clouds in their upper atmospheres. The case of TrES-3 is borderline, with an effective temperature of $1643 f^{1 / 4}(1-A)^{1 / 4} \mathrm{~K}$ (O'Donovan et al. 2007), where $A$ is the Bond albedo (the ratio of reflected to incident bolometric power) and $f$ is a phenomenological factor accounting for atmospheric circulation (with $f=1$ for isotropic re-radiation and $f=2$ for radiation from the day side only). For TrES-3 it would be possible to obtain a self-consistent Class $\mathrm{V}$ solution with highlyreflective silicate and iron clouds, e.g., $f=1$ and $A \approx 0.4$, giving $T_{\text {eff }}=1500 \mathrm{~K}$. Our upper limit on $p_{i}$ rules this out, and hence may be regarded as evidence against the presence of highly reflective clouds in the planet's upper atmosphere.

For cloud-free atmospheres, the thermal radiation from the planet is expected to produce a larger signal than the reflected light (see, e.g., López-Morales \& Seager 2007; Fortney et al. 2007). One might wonder whether our limits on the planetto-star flux ratio also constrain the thermal emission spectrum of the planet. The thermally emitted flux can be estimated from the effective temperature of the planet. For TrES-3, even for the hottest possible temperature of $2100 \mathrm{~K}$ (for $f=2$ and $A=0$ ) we estimate that the maximum planet-to-star flux ratio due solely to thermal emission is $2 \times 10^{-4}$ at a wavelength of $0.9 \mu \mathrm{m}$. This rough estimate agrees within a factor of 2 with a more detailed atmospheric model of TrES-3 by Fortney et al. (2007). Those investigators classify TrES-3 as a pM planet, ${ }^{10}$ for which they predict a very low albedo (because conditions are too hot for any condensates) and an "anomalously hot" brightness temperature (because of a high-altitude temperature inversion). For our observing bandpass the calculated planet-to-star flux ratio is $\sim 10^{-4}$. This is smaller than our most constraining upper limit of $2.4 \times 10^{-4}$ in the $i$-band, and hence we conclude that the expected level of thermal emission is beneath our detection limit by a factor of $2-3$.

In summary, through high-precision photometry of an especially favorable target, we have placed meaningful upper limits on the albedo of a transiting exoplanet. This is the first time this has been done with ground-based occultation photometry.

\footnotetext{
10 The distinction between $\mathrm{pM}$ and $\mathrm{pL}$ planets, in the nomenclature proposed by Fortney et al. (2007), is analogous to the $\mathrm{M} / \mathrm{L}$ transition in low-mass stars and brown dwarfs. The $\mathrm{pM}$ planets are hot enough for $\mathrm{TiO}$ and $\mathrm{VO}$ to exist in gaseous form, leading to strong absorption of the stellar flux at low pressure, a temperature inversion in the planet's upper atmosphere, and a brightness temperature in excess of the equilibrium temperature in the optical and infrared bands.
}

Our results seem to be limited by random noise, suggesting that the acquisition of more data will lead to increased sensitivity. For example, with 8 more light curves comparable in quality to the two $i$-band light curves presented here, the photon-limited $1 \sigma$ error in the albedo would be approximately 0.03 . Even if the albedo proves to be very small, as expected in cloud-free atmospheric models, such a data set would enable a $\sim 4 \sigma$ detection of the expected level of thermal emission. Of course, it is possible that sources of systematic noise will become limiting factors, such as flat-fielding errors, or time-variable differential extinction beyond a gradual air-mass dependence. Nevertheless, our results give reason to hope for an unambiguous detection of reflected light or thermal emission from exoplanets based on ground-based photometry using meter-class telescopes.

We thank Jonathan Fortney, Willie Torres, and Alex Sozzetti for helpful conversations and correspondence; John Southworth, for his code for computing limb-darkening coefficients; and an anonymous referee for a timely and detailed review. This research was supported by Grant No. 2006234 from the United States-Israel Binational Science Foundation (BSF), Jerusalem, Israel. KeplerCam was developed with partial support from the Kepler mission under NASA Cooperative Agreement NCC21390 (PI: D. Latham).

\section{REFERENCES}

Barman, T. S., Hauschildt, P. H., \& Allard, F. 2001, ApJ, 556, 885

Berdyugina, S. V., Berdyugin, A. V., Fluri, D. M., \& Piirola, V. 2008, ApJ, 673, L83

Charbonneau, D., Noyes, R. W., Korzennik, S. G., Nisenson, P., Jha, S., Vogt, S. S., \& Kibrick, R. I. 1999, ApJ, 522, L145

Claret, A. 2000, A\&A, 363, 1081

Claret, A. 2004, A\&A, 428, 1001

Cameron, A. C., Horne, K., Penny, A., \& James, D. 1999, Nature, 402, 751

Cameron, A. C., Horne, K., Penny, A., \& Leigh, C. 2002, MNRAS, 330, 187

Fortney, J. J., Lodders, K., Marley, M. S., \& Freedman, R. S. 2007, arXiv:0710.2558

Guillot, T., Burrows, A., Hubbard, W. B., Lunine, J. I., \& Saumon, D. 1996, ApJ, 459, L35

Holman, M. J., et al. 2007, ApJ, 664, 1185

Hough, J. H., Lucas, P. W., Bailey, J. A., Tamura, M., Hirst, E., Harrison, D., \& Bartholomew-Biggs, M. 2006, PASP, 118, 1302

Hubeny, I., Burrows, A., \& Sudarsky, D. 2003, ApJ, 594, 1011

Irwin, J., et al. 2008, arXiv:0801.1496

Karkoschka, E. 1994, Icarus, 111, 174

Leigh, C., Cameron, A. C., Horne, K., Penny, A., \& James, D. 2003b, MNRAS, 344, 1271

Leigh, C., Cameron, A. C., Udry, S., Donati, J.-F., Horne, K., James, D., \& Penny, A. 2003a, MNRAS, 346, L16

Liu, X., et al. 2008, arXiv:0711.2304

López-Morales, M., \& Seager, S. 2007, ApJ, 667, L191

Mandel, K., \& Agol, E. 2002, ApJ, 580, L171

Marley, M. S., Gelino, C., Stephens, D., Lunine, J. I., \& Freedman, R. 1999, ApJ, 513,879

O’Donovan, F. T., et al. 2007, ApJ, 663, L37

Rasio, F. A., Tout, C. A., Lubow, S. H., \& Livio, M. 1996, ApJ, 470, 1187

Rowe, J. F., et al. 2007, arXiv:0711.4111

Saumon, D., Hubbard, W. B., Burrows, A., Guillot, T., Lunine, J. I., \& Chabrier, G. 1996, ApJ, 460, 993

Seager, S., \& Sasselov, D. D. 1998, ApJ, 502, L157

Seager, S., Whitney, B. A., \& Sasselov, D. D. 2000, ApJ, 540, 504

Sharp, C. M., \& Burrows, A. 2007, ApJS, 168, 140

Southworth, J. 2008, arXiv:0802.3764

Sudarsky, D., Burrows, A., \& Hubeny, I. 2003, ApJ, 588, 1121

Sudarsky, D., Burrows, A., \& Pinto, P. 2000, ApJ, 538, 885

Szentgyorgyi, A. H., et al. 2005, BAAS, 37, 1339

Torres, G., Winn, J. N., \& Holman, M. J. 2008, arXiv:0801.1841

Winn, J. N., et al. 2007, AJ, 134, 1707

Young, A. T. 1967, AJ, 72, 747 